\title{
Residual cardiovascular risk in patients who received lipid-lowering treatment in a real-life setting: retrospective study
}

This article was published in the following Dove Press journal:

ClinicoEconomics and Outcomes Research

25 October 2016

Number of times this article has been viewed

\section{Valentina Perrone \\ Diego Sangiorgi \\ Stefano Buda \\ Luca Degli Esposti}

CliCon S.r.l., Health, Economics \&

Outcomes Research, Ravenna, Italy
Correspondence: Luca Degli Esposti

CliCon S.r.l., Health, Economics

\& Outcomes Research, Via Salara,

36 - 48100 Ravenna, Italy

Tel +3954438393

Fax +39544212699

Email luca.degliesposti@clicon.it
Introduction: The objective of this study was twofold: 1) to assess the residual cardiovascular (CV) risk among patients treated with statins according to guidelines and at the recommended dosages; and 2) to assess the difference, if any, in the frequency of CV events when patients were treated with other lipid-lowering agents alongside statins.

Methods: A retrospective observational study including one local health unit was conducted. Administrative databases were linked to laboratory test database in order to collect cholesterol values at baseline. Patients were included if they had filled at least one prescription for statins between January 1, 2009 and December 31, 2011; patients' records were considered for a 12-month time span.

Results: A total of 27,330 patients treated with statins were included (50\% male, mean age $68.0 \pm 11.5$ years). Among them, 770 were treated with statins according to guidelines and at the recommended dosages and had a low density lipoprotein-cholesterol value below the therapeutic target. Nevertheless, the risk of myocardial infarction or stroke remained: incidence rates were $1.3 \pm 1.0$ per patient per year for moderate $C V$ risk, $4.1 \pm 2.6$ for high risk, and $12.5 \pm 11.0$ for very high risk. This incremental risk was confirmed further using the Cox model, by correcting for age, sex, use of antiplatelet and/or antihypertensive therapy, and adherence to treatment. As a second analysis, we compared, after a propensity score matching, patients extracted from the overall sample who were treated with fibrates. Based on the Cox model, patients on fibrates had a risk for myocardial infarction or stroke lower than patients on statins.

Conclusion: Among patients treated with statins according to guidelines and at the recommended dosages, a residual CV risk was observed. We concluded that intervention for managing residual CV risk during statin therapy should be implemented.

Keywords: lipid lowering treatment, real-world data, residual cardiovascular risk

\section{Introduction}

Cardiovascular disease (CVD) is an important global public health problem that is associated with adverse health outcomes and high health care costs. ${ }^{1}$ CVD is a major cause of mortality and morbidity worldwide; in Europe, it accounts for over 4 million deaths each year. ${ }^{2}$ The guidelines for the prevention of CVD consider this disease as the product of several risk factors, such that when properly managed, CVD mortality can be reduced. 3,4

Many international guidelines recognize low-density lipoprotein cholesterol (LDL-C) as a primary target for lipid-lowering therapies. ${ }^{5}$ Statins are the first-line therapy for lowering LDL-C levels in blood; 5 studies have shown that whereas treatment with statins reduces the rate of cardiovascular (CV) events, it is not fully abated and a considerable 
residual risk remains even when achieving LDL-C levels at or below recommended targets. ${ }^{6,7}$

This is not due to failure in adherence to statin treatment. Studies in "real-world" populations and systematic reviews have shown that adherence to medication positively correlated with reduced CV risk, significantly improved health outcomes, and reduced annual costs; ${ }^{8,9}$ even in patients sufficiently compliant with statin treatment, a residual risk of about $69 \%$ persisted, yet; this incomplete reduction of risk might also result in ongoing progression of disease. ${ }^{7}$

When patients do not show an adequate response to statin therapy, the guidelines recommend increasing the dosage of statins or to combining statins with another lipid-lowering drug. ${ }^{10}$ The evidence for statin combination therapy in improving CV outcomes remains inconclusive. ${ }^{11}$

The aim of this study was to assess the residual CV risk among patients treated with statins according to guidelines and at the recommended dosages, and to assess the possible improvement in CV risk yielded by addition of another lipidlowering agent alongside statins.

\section{Methods}

\section{Data sources}

The study was based on administrative databases of one Italian local health unit (LHU), based in Emilia Romagna, which included $\sim 290,000$ health-assisted individuals.

In particular, the following databases were used: the health-assisted subjects' database, containing patients' demographic data; medications prescription databases, providing information for each medication prescription, such as the anatomical-therapeutic-chemical (ATC) code of the drug purchased; hospital discharge database, which includes all hospitalization data with the discharge diagnosis codes classified according to the International Classification of Diseases, Ninth Revision, Clinical Modification (ICD-9-CM); the clinical laboratory database, containing cholesterol value and the dates on which these were performed.

The patient code in each database permitted electronic linkage with all other databases. No identifiers related to patients were provided to the researchers. According to the Italian law for confidentiality of data, the study was notified to the local Ethics Committee of the LHU.

\section{Cohort definition}

This is a retrospective cohort study that includes all prescription and hospitalization data during the study period for statin users aged $\geq 18$ years.
Patients were enrolled if between January 1, 2009 and December 31, 2011 (enrolled period), they received one prescription for any statin drug (simvastatin [ATC code: C10AA01], lovastatin [ATC code: C10AA02], pravastatin [ATC code: C10AA03], fluvastatin [ATC code: C10AA04], atorvastatin [ATC code: C10AA05], rosuvastatin [ATC code: C10AA07], or simvastatin and ezetimibe [ATC code: C10BA02]). The date of first prescription of statin was identified as the "index-date", which represents the enrollment day of each individual patient, who was then followed for 1 year ("follow-up period"). Patients who were transferred to another LHU during the follow-up period (1 year to start enrolled data) were excluded from analysis.

\section{Patient characteristics}

The clinical characteristics and all prescription drugs of the patients enrolled in this study were investigated in the 1-year period before the index-date (characterization period).

Patients were classified as at high CV risk if they received treatment or hospitalization for diabetes (defined as at least two prescriptions of antidiabetic drugs [ATC code: A10] or at least one hospitalization with a diagnosis of diabetes [ICD-9-CM code: 250]) or had a traceable record of CV risk (previous hospitalization for acute cardiac ischemia [ICD9-CM code: 411], angina pectoris [ICD-9-CM code: 413], chronic cardiac ischemia [ICD-9-CM code: 414]; cerebral hemorrhage [ICD-9-CM code: 431]; cerebral artery occlusion [ICD-9-CM code: 434]; transient cerebral ischemia [ICD-9-CM code: 435]; cerebral circulatory disorders [ICD9-CM code: 436]; atherosclerosis [ICD-9-CM code: 440]; other peripheral vascular diseases [ICD-9-CM code: 443], or were treated with aspirin [ATC code: B01AC06] associated with beta-blockers [ATC code: C07] and/or ACE inhibitors [ATC codes: C09A, C09B]). Patients were classified as at very high $\mathrm{CV}$ risk if they had diabetes associated with coronary heart disease (previous hospitalization for acute cardiac ischemia and/or chronic coronary angioplasty [ICD-9-CM code: 0066,360$]$ ) or myocardial infarction (ICD-9-CM code: 410) or old myocardial infarction (ICD-9-CM code: 412). Patients were classified as at low/moderate CV risk on the basis of absence of claims with all ICD-9-CM codes used to define high and very high CV patients.

Patients in the analysis were characterized both for their baseline LDL-C levels, defined as the last value of cholesterol available in the 6 months before the index-date, and for the LDL-C level in the 6 months before the date of end of observation period (CV event or end of follow-up). Such measurements are needed both for classifying each patient 
based on the need for reduction of the cholesterol level, and to evaluate the achievement and not the therapeutic target. The distance from target was consequently defined as baseline LDL-C - LDL-C target/LDL-C at baseline $\times 100$.

The therapeutic target of LDL-C was set at $70 \mathrm{mg} / \mathrm{dL}$ for very high-risk patients, $100 \mathrm{mg} / \mathrm{dL}$ for high-risk patients, and $130 \mathrm{mg} / \mathrm{dL}$ for moderate-risk patients.

Based on current national guidelines for the management of dyslipidemia, patients were characterized on the basis of the prescribed dosage and statin, assessing the ability to achieve the target lipid in relation to the level of LDL at baseline. ${ }^{12}$

In order to evaluate the residual $\mathrm{CV}$ risk, we considered only patients with LDL-C levels at baseline and undergoing appropriate pharmacological treatment (statin and dosage adjusted to the initial level of LDL-C and adhering to treatment).

The patients were also characterized based on treatment with fibrates at baseline (ATC code: $\mathrm{C} 10 \mathrm{AB})$, antihypertensive drugs (ATC codes: C02, C03, C07, C08, C09), or antiplatelet agents (ATC code: B01). During the follow-up period, the first hospitalization for $\mathrm{CV}$ reasons (ICD-9-CM codes: 410, 411, 413, 414) or stroke (ICD-9-CM codes: 431, 434) was also evaluated.

\section{Assessment of adherence}

Adherence to statin therapy was estimated by calculating the proportion of days covered according to the method used by Catalan and LeLorier. ${ }^{13}$ A treatment episode was measured as the time span between the starting day of the first statin dispensation until the end day of the supply for the last statin dispensation. The latter included a permissible gap after the final dispensing within the specific episode. Patients were defined as adherent if $\geq 80 \%$ of the follow-up period was covered by drug dispensation.

\section{Statistical analysis}

Continuous variables are given as means with standard deviations; categorical variables are shown as percentages and absolute numbers. Student's $t$-test and chi-square tests were used to compare continuous and categorical values.

The determination of the outcomes was based on the incidence rates as events per 100 person/year and their confidence intervals at 95\%; we also used a multivariate model to Cox proportional hazards; Schoenfeld residual analysis was used to validate the proportional hazards assumption.

To minimize selection bias, survival analyses of multivariate comparison between patients with or without the addition of fibrates were carried out using a pairing 1:1 through propensity score. The propensity score was calculated using a logistic regression model that takes into account the probability of being treated with fibrates according to the characteristics at baseline for each patient (sex, age, previous treatment with antihypertensive and/or antiplatelet drugs, adherence to statins, and level of $\mathrm{CV}$ risk).

The Hosmer-Lemeshow and the C-statistic tests were used to evaluate the calibration and the discriminatory power of the model. The patients were then matched 1:1 within each quintile of propensity score. $P$-values $<0.05$ were considered statistically significant.

All statistical analyses were performed using the STATA software version 12.0 (Stata Corporation, College Station, TX, USA).

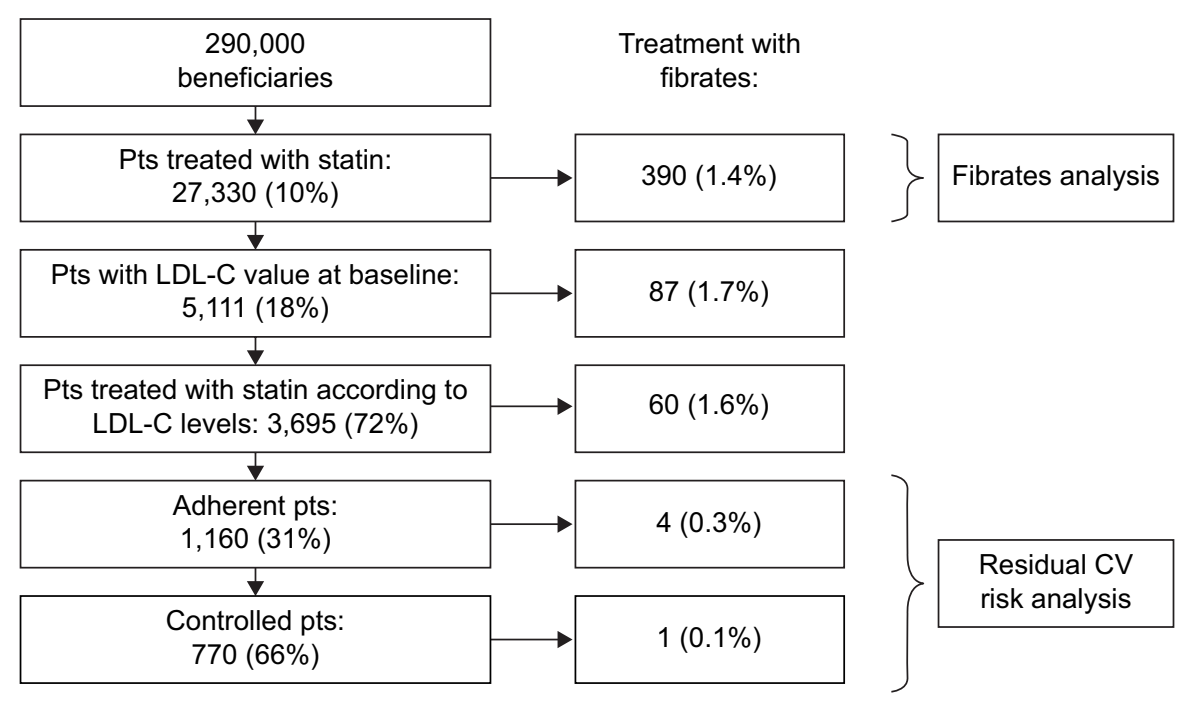

Figure I Flowchart of patients in analysis.

Abbreviations: CV, cardiovascular; LDL-C, low-density lipoprotein; Pts, patients. 


\section{Results}

The patients in analysis are summarized in the flowchart shown in Figure 1. A total of 27,330 patients treated with statin were included ( $10 \%$ of the enrolled patient population), with a mean age of $68.0 \pm 11.5$ years, of whom 13,690 were males $(50 \%)$ (Table 1$)$. In all patients included in this study, the CV risk was 55\%, 34\%, and $11 \%$ for levels of moderate, high, and very high risk, respectively.

Among the patients, 5,111 presented a LDL-C measurement at baseline (18\% of the total); of whom 3,695 (72\% of patients with a LDL-C measurement) were also treated with a statin and dosage adjusted to reach LDL-C in the range accepted as therapeutic target (Figure 1).

A total of 1,160 patients were adherent to statins and reached the target value as recommended in the guidelines in routine clinical practice (Table 2 ); $55 \%$ were males and the mean age was $68.1 \pm 10.5$ years. The CV risk was found to be moderate in $52 \%$ of cases, high in $35 \%$, and very high in $13 \%$ (Table 2).

Despite these patients using the drug appropriately, there was an increase in the number of $\mathrm{CV}$ events with increasing level of CV risk: incidence rates were $1.0 \pm 0.8,4.3 \pm 2.1$, and 9.6 \pm 5.2 events per 100 person/year for levels of moderate, high, and very high risk, respectively (Figure 2). For descriptive purposes, we considered nonadherent patients treated with statin and at an appropriate dosage; incidence rates were $3.8 \pm 0.9,10.5 \pm 2.6$, and $69.8 \pm 15.9$ events per 100 persons/year for levels of moderate, high, and very high risk, respectively (Figure 2).

Table I Characteristics of the study population

\begin{tabular}{ll}
\hline & Total \\
\hline $\mathrm{N}$ & 27,330 \\
Male, $\mathrm{n}(\%)$ & $13,690(50)$ \\
Age (years) & $68.0 \pm \mathrm{I} \mathrm{I} .5$ \\
CV risk, n (\%) & \\
$\quad$ Moderate & $15,132(55)$ \\
High & $9,210(34)$ \\
$\quad$ Very high & $2,988(11)$ \\
Adherent & $7,998(30)$ \\
\hline
\end{tabular}

Abbreviation: CV, cardiovascular.

Table 2 Characteristic of adherent and controlled patients

\begin{tabular}{lll}
\hline & Adherent & Adherent and controlled \\
\hline $\mathrm{N}$ & $\mathrm{I}, 160$ & 770 \\
Male, $\mathrm{n}(\%)$ & $648(55)$ & $428(55)$ \\
Age (years) & $68.1 \pm 10.5$ & $68.0 \pm 10.0$ \\
$\mathrm{CV}$ risk, $\mathrm{n}(\%)$ & & \\
$\quad$ Moderate & $603(52)$ & $474(62)$ \\
$\quad$ High & $409(35)$ & $25 \mathrm{I}(33)$ \\
$\quad$ Very high & $148(13)$ & $45(6)$ \\
\hline
\end{tabular}

Abbreviation: $\mathrm{CV}$, cardiovascular.

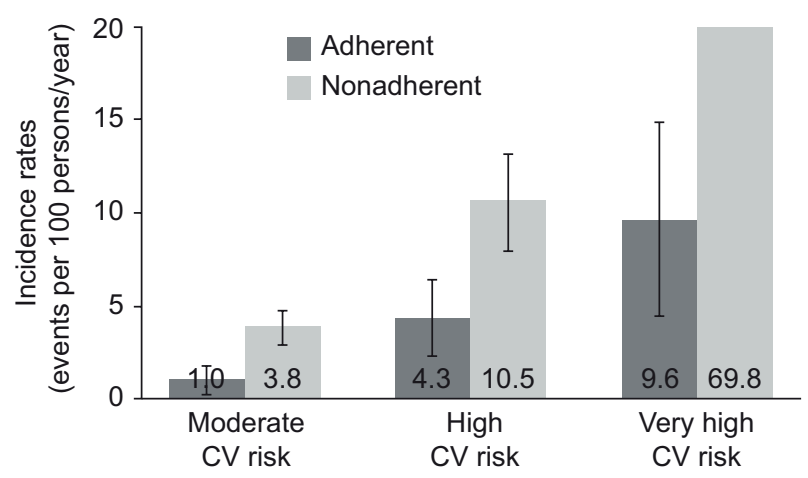

Figure 2 Incidence of CV events according to the level of risk among adherent and nonadherent patients.

Notes: Among adherent patients, the incidence rates were: $1.0 \pm 0.8,4.3 \pm 2.1$, and $9.6 \pm 5.2$ events per 100 person/year for levels of moderate, high, and very high risk, respectively. Among nonadherent patients, the incidence rates were $3.8 \pm 0.9$, $10.5 \pm 2.6$, and $69.8 \pm 15.9$ events per 100 persons/year for levels of moderate, high, and very high risk, respectively.

Abbreviation: CV, cardiovascular.

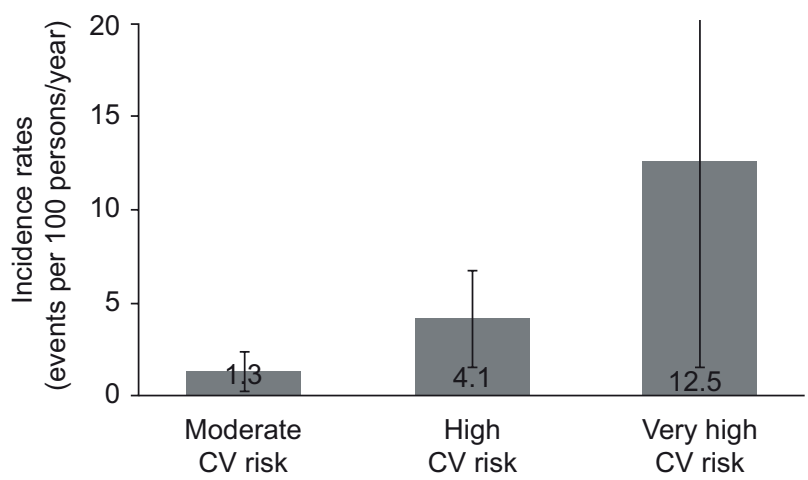

Figure 3 Incidence of $\mathrm{CV}$ events according to the level of risk among controlled patients.

Notes: Incidence rates were $1.3 \pm 1.0,4.1 \pm 2.6,12.5 \pm 11.0$ events, respectively, per 100 persons/year for levels of moderate, high, and very high risk, respectively Abbreviation: $\mathrm{CV}$, cardiovascular.

From this adherent and controlled group, 770 patients were extracted who had achieved the lipid target $(66 \%$ of patients with LDL-C measured at baseline and with an appropriate drug treatment) with a mean age of $68.0 \pm 10.0$ years, 428 patients (55\%) were males (Table 2 ). The baseline characteristics of adherent patients and adherent patients who achieved the lipid target appeared to be comparable with however a slightly lower $\mathrm{CV}$ risk: moderate in $62 \%$ of cases, high in $33 \%$, and very high in $6 \%$ (Table 2 ), with incidence rates of $1.3 \pm 1.0,4.1 \pm 2.6,12.5 \pm 11.0$ events, respectively, per 100 persons/year (Figure 3).

An analysis of both groups using the multivariate Cox model revealed for adherent patients a 2.5 -fold increment in risk in high- versus moderate-risk patients and fivefold increment in risk in very high- versus moderate-risk patients. Sex analysis revealed that the risk of CV events or stroke was 2.5 -fold higher in males than females. We observed similar results for adherent patients who achieved the lipid 
Table 3 Multivariate model regression

\begin{tabular}{|c|c|c|c|c|c|c|c|}
\hline & \multicolumn{3}{|c|}{ Adherent } & \multicolumn{4}{|c|}{ Adherent and controlled } \\
\hline & HR & $95 \% \mathrm{Cl}$ & $P$ & $\mathbf{H R}$ & $95 \% \mathrm{Cl}$ & & $\boldsymbol{P}$ \\
\hline Male & 2.643 & $1.126,6.207$ & 0.026 & 2.516 & 0.813 & 7.790 & 0.110 \\
\hline Age (years) & 1.017 & $0.98 \mathrm{I}, \mathrm{I} .054$ & 0.356 & 1.006 & 0.961 & 1.053 & 0.803 \\
\hline \multicolumn{8}{|l|}{$\mathrm{CV}$ risk } \\
\hline Moderate & REF & & & REF & & & \\
\hline High & 2.570 & $0.933,7.081$ & 0.068 & 1.914 & 0.628 & 5.831 & 0.254 \\
\hline Very high & 5.135 & $1.795,14.692$ & 0.002 & 4.969 & 1.358 & 18.178 & 0.015 \\
\hline Antihypertensive drugs at baseline & 5.639 & $0.721,44.084$ & 0.099 & - & - & - & - \\
\hline Antiplatelet drugs at baseline & 1.278 & $0.438,3.729$ & 0.654 & 1.215 & 0.347 & 4.247 & 0.761 \\
\hline
\end{tabular}

Note: Adherent and controlled patients.

Abbreviations: $\mathrm{Cl}$, confidence interval; $\mathrm{CV}$, cardiovascular; $\mathrm{HR}$, hazard ratio; REF, reference.

Table 4 Characteristics of the study population (statin alone and statin plus fenofibrate)

\begin{tabular}{|c|c|c|c|c|c|}
\hline & No fibrates & Fibrates & $P$ & $\begin{array}{l}\text { No fibrates, } \\
\text { pairing } I: I\end{array}$ & $P$ \\
\hline N (\%) & $26,940(98.6)$ & $390(1.4)$ & & 390 & \\
\hline Male, n (\%) & $|3,49|(50)$ & $199(5 \mathrm{I})$ & 0.743 & $200(5 \mathrm{I})$ & 1.000 \\
\hline Age (years) & $68.0 \pm 11.5$ & $64.6 \pm 11.3$ & $<0.001$ & $64.4 \pm 11.7$ & 0.808 \\
\hline CV risk, n (\%) & & & 0.002 & & 0.446 \\
\hline Moderate & $14,885(55)$ & $247(63)$ & & $243(62)$ & \\
\hline High & $9,093(34)$ & 117 (30) & & $128(33)$ & \\
\hline Very high & $2,962(\mathrm{II})$ & $26(7)$ & & $19(5)$ & \\
\hline Adherent & $7,960(30)$ & $38(10)$ & $<0.001$ & $44(\mathrm{II})$ & 0.559 \\
\hline
\end{tabular}

Abbreviation: $\mathrm{CV}$, cardiovascular.

target, a twofold increment in risk in patients at high versus moderate risk (despite not reaching statistical significance, due to the small number of patients in this risk class) and fivefold increment in risk in patients at very high risk versus moderate risk (Table 3 ).

Within the overall group of patients receiving statin therapy, 390 patients (1.4\%) at baseline took fibrates in addition to statins (Figure 1); of these, the main age was $64.6 \pm 11.3$ years and $51 \%$ of patients were male (Table 4 ). Among patients treated with fibrates, we observed a slightly lower CV risk, with percentages of $30 \%$ and $7 \%$ for levels of high and very high risk, respectively. In terms of adherence to the therapy, the group of patients treated with fibrates showed levels lower than those of patients on statins only, with a percentage of adherent patients at $10 \%$, against $30 \%$ of the total sample; after pairing via propensity score, these differences were, however no longer observed (Table 4).

An analysis with the multivariable Cox model showed that positively related to the risk of a $\mathrm{CV}$ event or stroke were the following covariates: male (with an increase in risk of 3.7 versus females), very high CV risk (with an increase in risk of 3.1 versus moderate $\mathrm{CV}$ risk), age (with a risk increase of $4 \%$ for each year of life), and use of fibrates with a risk
Table 5 Multivariate model regression

\begin{tabular}{lllll}
\hline & HR & 95\% Cl & P \\
\hline Male & 3.704 & 1.638 & 8.377 & 0.002 \\
Age (years) & 1.038 & 1.004 & 1.072 & 0.028 \\
CV risk & & & & \\
$\quad$ Moderate & & & & \\
$\quad$ High & 1.414 & 0.600 & 3.330 & 0.428 \\
$\quad$ Very high & 3.123 & 1.009 & 9.663 & 0.048 \\
Fibrate & 0.452 & 0.224 & 0.912 & 0.027 \\
Adherence >80\% & 0.326 & 0.077 & 1.386 & 0.129 \\
Antihypertensive drugs at baseline & 1.349 & 0.508 & 3.584 & 0.548 \\
Antiplatelet drugs at baseline & 0.943 & 0.390 & 2.284 & 0.897 \\
\hline
\end{tabular}

Note: Patients in treatment with fenofibrate and statin.

Abbreviations: $\mathrm{Cl}$, confidence interval; $\mathrm{CV}$, cardiovascular; $\mathrm{HR}$, hazard ratio.

reduction (hazard ratio $=0.452$ [confidence interval $95 \% 0.22$, 0.91], $P=0.048$ ) (Table 5).

During the follow-up period, 1,774 CV events (6.5-7.2 per 100 persons/year) occurred among the group of patients treated with statins and $12 \mathrm{CV}$ events (1.4-4.9 per 100 persons/year) among the group of patients treated with statins and fibrates, respectively.

\section{Discussion}

This retrospective analysis in a "real-world" setting showed that about $10 \%$ of all patients in the study were users of statin therapy. The European Society of Cardiology has shown that the use of lipid-lowering drugs has massively increased in patients diagnosed with $\mathrm{CV}$ conditions. ${ }^{2,14}$ According to previous studies, statins are used extensively in most European countries, with an average annual increase in the rate of usage of $31 \% .^{15,16}$ In Italy, the rate has more than tripled, increasing from $25 \%$ of patients in $1995 / 1996$ to $91 \%$ of patients in 2006/2007. ${ }^{2}$

According to guidelines and recommendations for dyslipidemia treatment, statin therapy should be initiated according to an individual's risk of CVD, and based on LDL-C baseline levels and the defined LDL-C target level. Despite the 
efficacy of the current statin-based therapies, several studies have observed that many patients treated with statins do not reach the therapeutic goal while remaining at risk of future CV events. ${ }^{17}$

Our results are in agreement with these previous analyses, as they show that the incremental risk of CV events increased in high-risk patients despite statins having been prescribed in agreement with guidelines and patients adhering to therapy.

Our results also show that, in patients who discontinued treatment, the risk of $\mathrm{CV}$ events was higher than in patients adherent to treatment. This is in line with the results of a number of studies that analyzed the effect of adherence to statin treatment and health outcomes. ${ }^{8,18-20}$ A recent Italian retrospective analysis estimated that suboptimal adherence to statins occurred in a substantial proportion of newly treated patients, and was associated with increased risk of adverse health outcomes. ${ }^{21}$ This suggests that interventions to improve adherence, especially in chronic conditions, need to be broadly applied.

Another important finding in this study is the observation that patients who achieve a target for LDL-C, in the setting of optimal LDL-C reduction, had a risk of CV events lower than patients who did not achieve these goals. Serum lipid goal achievement in patients included in this study was based on the most recent European Society of Cardiology/European Atherosclerosis Society guidelines for the management of dyslipidemias. $^{5}$

The residual $\mathrm{CV}$ risk may in part be attributable to low high-density lipoprotein cholesterol, high triglyceride levels, and patients' characteristics such as genetic traits and behaviors. ${ }^{7}$ Several studies have shown that increasing high-density lipoprotein cholesterol and reducing the concentration of triglycerides is a valuable strategy to address this residual risk. Indeed, it has been reported that triglycerides were reduced further or even eliminated by approaches that involved the addition of a concomitant lipid-lowering therapy with a mechanism of action additive to that of statins. ${ }^{22,23}$ Combined fibrate/statin therapy is the treatment of choice as it is effective in achieving comprehensive lipid control and may lead to additional CV risk reduction. ${ }^{24-26}$

In our study, the sample of patients receiving a combined fibrate/statin therapy was small. Despite this, a multivariate analysis indicated that these patients had a reduced risk of $\mathrm{CV}$ events. The evidence for the combined fibrate/statin therapy in improving CV outcomes in dyslipidemic patients while still inconclusive is encouraging further studies; in particular, the efficacy of therapies combining statins with fibrates, niacin, bile acid sequestrants, and ezetimibe in patients at high CVD risk, as well as potential new treatments, including new lipid-modifying drugs currently under investigation or recently approved should be investigated, and in a real-life setting.

Our results should be interpreted in the context of the several limitations of this study. First, the primary limitation is the observational nature of the study design. Second, shared by other similar observational studies, the absence of the relevant clinical information in the data setting. The severity of disease or the reasons for no adherence of treatment of the patients are not retrievable from the dataset. In addition, our study did not collect data related to patient lifestyle (as information on smoking), genetic predisposition to CVD, or the presence of other risk factors likely to modulate the risk of a new event; these factors, could act as confounders of the association between statin therapy and $\mathrm{CV}$ outcomes. In general, administrative database analyses limit the interpretation of results depending on the information available. Third, the small sample size in fact, the analyses were performed using the databases of one LHU; hence the results of this study have to be interpreted with caution and further larger studies are required to confirm these results. Fourth, the relatively small sample size for patients in treatment with fibrates and the lack of LDL-C levels for these patients. Finally, the out-of-hospital death events are not retrievable from the dataset.

Our findings nevertheless provide evidence for residual $\mathrm{CV}$ risk despite statin-induced optimal LDL-C reduction per existing treatment guidelines. In these circumstances, since the origin of residual risk is multifactorial, the adoption of individual patient management should be considered as a serious option to reach therapeutic goals.

\section{Disclosure}

The authors report no conflicts of interest in this work.

\section{References}

1. GBD 2013 Mortality and Causes of Death Collaborators. Global, regional, and national age-sex specific all-cause and cause-specific mortality for 240 causes of death, 1990-2013: a systematic analysis for the global burden of disease study 2013. Lancet. 2015;385(9963): 117-171.

2. Nichols M, Townsend N, Luengo-Fernandez R, et al. European Cardiovascular Disease Statistics 2012. Brussels: European Heart Network, Sophia Antipolis: European Society of Cardiology; 2012.

3. Mills EJ, Rachlis B, Wu P, Devereaux PJ, Arora P, Perri D. Primary prevention of cardiovascular mortality and events with statin treatments: a network meta-analysis involving more than 65,000 patients. J Am Coll Cardiol. 2008;52(22):1769-1781.

4. Anderson TJ, Gregoire J, Hegele RA, et al. 2012 update of the Canadian Cardiovascular Society guidelines for the diagnosis and treatment of dyslipidemia for the prevention of cardiovascular disease in the adult. Can J Cardiol. 2013;29(2):151-167.

5. Flink L, Underberg JA, Newman JD, Gianos E. The recent national lipid association recommendations: how do they compare to other established dyslipidemia guidelines? Curr Atheroscler Rep. 2015;17(4):494. 
6. Sirimarco G, Labreuche J, Bruckert E, et al. Atherogenic dyslipidemia and residual cardiovascular risk in statin-treated patients. Stroke. 2014;45(5): 1429-1436.

7. Kones R. Molecular sources of residual cardiovascular risk, clinical signals, and innovative solutions: relationship with subclinical disease, undertreatment, and poor adherence: implications of new evidence upon optimizing cardiovascular patient outcomes. Vasc Health Risk Manag. 2013;9:617-670.

8. Bitton A, Choudhry NK, Matlin OS, Swanton K, Shrank WH. The impact of medication adherence on coronary artery disease costs and outcomes: a systematic review. Am J Med. 2013;126(4):357.e357-357.e327.

9. Rasmussen JN, Chong A, Alter DA. Relationship between adherence to evidence-based pharmacotherapy and long-term mortality after acute myocardial infarction. JAMA. 2007;297(2):177-186.

10. Bays HE, Jones PH, Brown WV, Jacobson TA. National lipid association annual summary of clinical lipidology 2015.JClin Lipidol. 2014;8(6 Suppl): S1-36.

11. Noto D, Cefalu AB, Averna MR. Beyond statins: new lipid lowering strategies to reduce cardiovascular risk. Curr Atheroscler Rep. 2014; $16(6): 414$

12. Guidelines on statin prescribing in the prevention of cardiovascular disease. NHS Foundation Trust 2006.

13. Catalan VS, LeLorier J. Predictors of long-term persistence on statins in a subsidized clinical population. Value Health. 2000;3(6):417-426.

14. Kotseva K, Wood D, De Bacquer D, et al. EUROASPIRE IV: A European Society of Cardiology survey on the lifestyle, risk factor and therapeutic management of coronary patients from 24 European countries. Eur $J$ Prev Cardiol. 2015;23(6):636-648.

15. Walley T, Folino-Gallo P, Schwabe U, van Ganse E. Variations and increase in use of statins across Europe: data from administrative databases. BMJ. 2004;328(7436):385-386.

16. Alnouri F, Wood D, Kotseva K, Ibrahim ME. Which statin worked best to achieve lipid level targets in a European registry? A post-hoc analysis of the EUROASPIRE III for coronary heart disease patients. J Saudi Heart Assoc. 2014;26(4):183-191.
17. Sampson UK, Fazio S, Linton MF. Residual cardiovascular risk despite optimal LDL cholesterol reduction with statins: the evidence, etiology, and therapeutic challenges. Curr Atheroscler Rep. 2012;14(1):1-10.

18. Volpe M, Degli Esposti L, Romeo F, et al. Il ruolo dell'aderenza al trattamento farmacologico nella terapia cronica delle malattie cardiovascolari: documento intersocietario di consenso. [Role of adherence to long-term drug therapy in patients with cardiovascular disease: an Italian intersocietary consensus document]. G Ital Cardiol (Rome). 2014;15(10 Suppl 1): $3 \mathrm{~s}-10 \mathrm{~s}$.

19. Naderi SH, Bestwick JP, Wald DS. Adherence to drugs that prevent cardiovascular disease: meta-analysis on 376,162 patients. Am JMed. 2012; 125(9):882-887.e881.

20. Latry P, Molimard M, Dedieu B, Couffinhal T, Begaud B, Martin-Latry K. Adherence with statins in a real-life setting is better when associated cardiovascular risk factors increase: a cohort study. BMC Cardiovasc Disord. 2011;11:46.

21. Degli Esposti L, Saragoni S, Batacchi P, et al. Adherence to statin treatment and health outcomes in an Italian cohort of newly treated patients: results from an administrative database analysis. Clin Ther. 2012;34(1) 190-199.

22. Norata GD, Ballantyne CM, Catapano AL. New therapeutic principles in dyslipidaemia: focus on LDL and Lp(a) lowering drugs. Eur Heart J. 2013;34(24):1783-1789.

23. Catapano AL, Farnier M, Foody JM, et al. Combination therapy in dyslipidemia: where are we now?Atherosclerosis. 2014;237(1): 319-335.

24. Tziomalos K. Clinical controversies in lipid management. Panminerva Med. 2015;57(2):65-70

25. Bruckert E, Labreuche J, Deplanque D, Touboul PJ, Amarenco P. Fibrates effect on cardiovascular risk is greater in patients with high triglyceride levels or atherogenic dyslipidemia profile: a systematic review and meta-analysis. J Cardiovasc Pharmacol. 2011;57(2):267-272.

26. Tenenbaum A, Fisman EZ. Fibrates are an essential part of modern anti-dyslipidemic arsenal: spotlight on atherogenic dyslipidemia and residual risk reduction. Cardiovasc Diabetol. 2012;11:125.

\section{ClinicoEconomics and Outcomes Research}

\section{Publish your work in this journal}

ClinicoEconomics and Outcomes Research is an international, peerreviewed open-access journal focusing on health technology assessment, pharmacoeconomics and outcomes research in the areas of diagnosis, medical devices, and clinical, surgical and pharmacologica intervention. The economic impact of health policy and health systems organization also constitute important areas of coverage. The manuscript management system is completely online and includes a very quick and fair peer-review system, which is all easy to use. Visit http://www.dovepress.com/testimonials.php to read real quotes from published authors. 